\title{
Estudos funcionais dos componentes separados da hemoglobina de um bagre de respiração aérea, Hoplosternum littorale (Hancock)*
}

\author{
Robert L. Garlick ('); H. Franklin Bunn $\left({ }^{2}\right)$; Hans Jorgen Fyhn $\left({ }^{3}\right) ;$ Unni E. H. Fyhn $\left({ }^{3}\right)$; Joseph \\ P. Martin ( $\left.{ }^{4}\right)$; Robert W. Noble ${ }^{(5)}$; Dennis A Powers $\left(^{6}\right)$
}

\begin{abstract}
Resumo
Foram isoladas as duas hemoglobinas, I e II do bagre de respiraçăo aérea obrigatória Hoplosternum littorale. A hemoglobina não fracionada apresenta alta atividade de oxigênio, efeito Bohr alcalino normal e efeito Root. Tanto o efeito Bohr quanto o efeito Root são aumentados por $1 \mathrm{mM}$ ATP. A hemoglobina I fracionada apresenta uma atividade relativamente alta de oxigênio, um efeito Bohr inverso entre $\mathrm{pH} 6,0$ e 8,0 $(\Delta \log \mathrm{p} 50 / \Delta \mathrm{pH}>0)$, porém sem apresentar efeito Root. Adição de $1 \mathrm{mM}$ ATP à hemoglobina I causa uma redução acentuada na afinidade oxigênio, mudança a um efeito Bohr alcalino normal $(\Delta \log \mathrm{p} 50 / \Delta \mathrm{pH}>0)$ mas não no efeito Root. A hemoglobina II fracionada apresenta menor afinidade de oxigênio a $\mathrm{pH}$ baixo e maior afinidade de oxigênio a $\mathrm{pH}$ alto que a hemoglobina I. A hemoglobina II apresenta um efeito Bohr alcalino acentuado que só é ligeiramente aumentado por $1 \mathrm{mM}$ ATP e um efeito Root a pH baixo que é aumentado por $1 \mathrm{mM}$ ATP. As velocidades de dissociação de $\mathrm{O}_{2}$ observadas e combinação de $\mathrm{CO}$ com os componentes I e II apresentam diferenças paralelas às observadas nas medições de equilibrio de oxigênio.
\end{abstract}

\section{INTRODUÇÃO}

Aguas doces tropicais de movimento lento são freqüentemente hipóxicas, quentes e podem apresentar altas concentrações de $\mathrm{CO}_{2}$. Estes fatores dificultam a respiração aquática e órgãos especiais, sangue e padrões de comportamento têm evoluído em certos peixes tropicais permitindo-lhes respirar oxigênio atmosférico (Carter \& Beadle, 1931; Johansen, 1970; Kramer \& Graham, 1976). O bagre Hoplostrnum littorale respira oxigênio atmosférico por meio de uma secção do intestino de paredes delgadas altamente vascularizada lo- calizada em seguida ao estômago. As paredes desta secção do intestino são aglandulares, não ciliadas e apresentam vasos sanguíneos grandes na parte externa e densas camadas capilares no interior. Esta parte respiratória ocupa a maior parte do intestino. Por dissecção tem-se mostrado desprovidas de alimentos e cheias de ar (Carter \& Beadle, 1931). H. littorale vai à superfície para respirar a cada 10 minutos independente da concentraçåo de $\mathrm{CO}_{2}$ da água e seu sangue apresenta uma capacidade de oxigênio muito alta relativa aos sangues de outros peixes das mesmas águas (Willmer, 1934). Concluímos que esta espécie, se mantida úmida, podia sobreviver fora da água durante a noite na ponte do "Alpha Helix".

Eletroforese preliminar (Fyhn et al., 1978) e experimentos de focalização, isoelétrica (Bunn \& Riggs, 1978) indicam que a hemoglobina de $\mathrm{H}$. littorale apresenta dois componentes principais, um dos quais apresenta um efeito Bohr alcalino e o outro com um efeito Bohr inverno. Neste trabalho, temos fracionado os dois componentes da hemoglobina de $H$. littorale e temos examinado os equilibrios de oxigênio e cinética de reaçōes com ligantes para cada componente.

\section{MATERIAIS E MÉTODOS}

Amostras de Haplosternum littorale foram obtidas em novembro e dezembro de 1976, durante a IV Expedição Amazônica do R/V “Alpha Helix", no rio Solimões, num local aproximadamente $30 \mathrm{~km}$ rio acima da união deste com

(*) - Versão original inglesa publicada em Comp. Blochem. Physiol. vol. 62A (1). 1979.

(1) - Department of Zoology, University of Texas, Austin, Texas, 78712, E.U.A.

(2) - Department of Medicine, Peter Bent Brigham Hospital, Havard Medical School, 721 Huntington, Avenue, Boston, Massachusetts, 02115, E.U.A.

(3) - Institute of Zoophysiology University of Oslo, Blindern, Oslo 3, Norway.

(4) - Duke University Marine Laboratory, Beuafort, North Carolina 28516, E.U.A.

(5) - Department of Medicine and Biochemistry, Veterans Administration Hospital, State University of New York, Buffalo, New York, 14215, E.U.A. Established Investigator of the American Heart Association.

(6) - Department of Biology, Johns Hupkins University Baltimore, Maryland, 21218, E.U.A. 
o rio Negro para formar o Amazonas. Os animais (peso corporal de $84-308 \mathrm{~g}$ ) foram tirados de uma pequena lagoa hipóxica perto do Lago de Janauacá ou foram comprados de um pescador local. Os peixes foram sangrados por punção cardíaca com uma seringa de vidro fria heparinizada e as células foram lavadas três vezes, em 10 volumes de $1,7 \% \mathrm{NaCl}, 1 \mathrm{mM}$ Tris $\mathrm{pH} 8,0$. As células foram logo lisadas por uma hora sobre gelo em 3 volumes de $1 \mathrm{mM}$ Tris, $\mathrm{pH} 8,0$. O lisado foi feito em $0,1 \mathrm{M} \mathrm{NaCl}$ e foi centrifugado a alta velocidade durante 15 minutos para remover estromas e resíduos celular.

Eletroforese em gel de disco de poliacrilamida $(\mathrm{pH} 8,9)$, registro de gel e eletroforese de gel de poliacrilamida de dodecil sulfato de sódio (SDS) foram realizadas como em Fyhn et al (1978). A relação entre a distância de migração de um componente da hemoglobina e a distância de migração de albumina de soro bovino em geis de disco alcalinos foi calculada e usada para comparar a mobilidade relativa dos componentes da hemoglobina.

A natureza do efeito Bohr de cada componente foi examinada analiticamente por focalização elétrico em gel de disco de poliacrilamida das deoxi e carbóxi hemoglobinas como descrito por Bunn \& Riggs (1978).

As curvas de equilibrio de oxigênio foram determinadas para sangue total usando $\sigma$ analisador de dissociação de Oxigênio Hem-OScan da Aminco (American Instrument Company, Silver Spring, Md) como descrito por powers et al. (1978). Amostras de sangue total foram deoxigenadas com nitrogênic $99,999 \%$ puro ou com nitrogênio contendo $5,6 \%$ de $\mathrm{CO}_{2}$ e foram reoxigenadas com ar contendo $21 \%$ de oxigênio ou com $69,4 \% \quad \mathrm{~N}_{2}$ $25 \% \mathrm{O}_{2}$ e $5,6 \% \mathrm{CO}_{2}$.

Os glóbulos vermelhos lisados foram destituídos de fosfatos orgânicos $e^{*}$ outros ions passando o lisado primeiro através de Sephadex G-25 médio (coluna de $2 \times 50 \mathrm{~cm}$ ) em Tris $0,1 \mathrm{mM}, \mathrm{pH} 8,5$ e logo através de uma coluna de $1,5 \times 30 \mathrm{~cm}$ formada pelas seguintes resinas de cima para baixo: $2 \mathrm{~cm}$ de Dowex-1 (em acetato), $2 \mathrm{~cm}$ Dowex-50w (forma de ion de amô. nio) e um leito de $20 \mathrm{~cm}$ de uma mistura intercambiadora de ions Bio Rad AG 501x8-D.
Os componentes da hemoglobina foram separados numa coluna de $3 \times 20 \mathrm{~cm}$ de DEAESephadex A-50 (Sigma Chem. Co.). Uma solução contendo $600 \mathrm{mg}$ de oxihemoglobina fracionada foi concentrada a $20 \mathrm{ml}$ num saco de diálise colocado numa suspensão de glicol de polietileno de alto peso molecular (peso médio, 20.000) e foi dialisada com mudanças freqüentes de Tris $50 \mathrm{mM}, \mathrm{pH} 8,5$ e colocada na coluna de DEAE-Sephadex mantendo equilíbrio com o mesmo tampão Tris, $700 \mathrm{ml}$ do tampão inicial foram passados pela coluna antes de ser estabelecido um gradiente linear entre $600 \mathrm{ml}$ de tampão Bis-tris de pH 7,0. A taxa de fluxo foi de ca. $35 \mathrm{ml} /$ hora. Fraçōes de $7,5 \mathrm{ml}$ foram coletadas e monitoradas manualmente a $542 \mathrm{~nm}$. As fraçōes separadas de hemoglobina foram logo reunidas e separadas novamente através da coluna G-25 e a coluna de fracionamento de intercâmbio iônico. Os componentes de hemoglobina separados foram usados para medições de equilíbrio de oxigênio, medições cinéticas e re-eletroforese. Todos os passos de purificação foram realizados à temperatura de $0-10^{\circ} \mathrm{C}$.

Componentes fracionados e hemoglobina foram diluídos com água destilada e tampōes de força iônica de $0,2(I)$ até uma concentração final de hemoglobina de $50 \mu \mathrm{M}$ (em base a heme) e força iônica igual a uma constante 0,051 . Os tampões foram preparados com Bistris a $\mathrm{pH}$ menores de 7,5 ou Tris a $\mathrm{pH}$ maiores que este valor. Os equilíbrios de oxigênio foram determinados espectofotometricamente em tonômetros de vidro a $20^{\circ} \mathrm{C}$ (Riggs \& Wolbach, 1956) a longitudes de onda de $558 \mathrm{e}$ $576 \mathrm{~nm}$. O valor do coeficiente de Hill, $n$, foi determinado a partir da inclinaçâo média dos resultados de Hill obtidos entre saturações de 25 e $75 \%$. Equilibrios de oxigênio foram determinados para hemoglobina fracionada e para hemoglobina mais adição de $1 \mathrm{ImM}$ de ATP. Para amostras a $\mathrm{pH}$ baixo nas quais a hemoglobina não podia ser saturada com o oxigênio atmosférico (dado $\sigma$ efeito Root) uma aliquota de Tris sólido foi introduzido no tonômetro para aumentar o $\mathrm{pH}$, o suficiente para poder saturar a hemoglobina completamente com $\mathrm{O}_{2}$. Este procedimento permitiu determinar o espectro da hemoglobina totalmente oxigenada. 
As curvas de transição do efeito Root foram calculadas diretamente a partir das curvas de equilíbrio de oxigênio ou foram determinadas da seguinte maneira. A hemoglobina foi diluída com Tris ou Bis-tris até um volume final de $3,0 \mathrm{ml}$ e uma concentração final de hemoglobina de $48 \mu \mathrm{M}$ (heme); registros de oxi e deoxihemoglobina foram determinados da mesma amostra. As amostras foram deoxige. nadas com uns poucos cristais de ditionito de sódio. Se uma amostra de $\mathrm{pH}$ baixo não estiver totalmente oxigenada a tensões atmosféricas de oxigênio, entäo suficiente Tris sódio foi adicionado à amostra para levá-la a um $\mathrm{pH}$ ao qual a hemoglobina estivesse saturada com oxigênio atmosférico.

Todas as mediçōes cinéticas foram realizadas com um aparelho "stopped-flow" do tipo originalmente descrito por Gibson \& Milnes (1964). Em todos os casos a força iônica da solução final após misturar foi de 0,05 . As constantes cinéticas apresentados são ajustados aos quadrados mínimos nos $65 \%$ iniciais de reação observada.

As cinéticas de dissociação de oxigênio foram medidas pelo processo de mudança de $\mathrm{pH}$ descrito por Noble et. al. (1970). Hemoglobina oxigenada em $1 \mathrm{mM}$ Tris de $\mathrm{pH} 8,0$ foi misturada com uma solução de ditionito de sódio em um tampão do pH desejado de força iônica de 0,1. A concentração final de hemoglobina foi aproximadamente de $30 \mu \mathrm{M}$ em hemoequivalentes e a reação foi seguida a 560 e $540 \mathrm{~nm}$.

As cinéticas de combinação de monóxido de carbono cum a hemoglobina foram medidas misturando as soluções de hemoglobina deoxigenada em tampōes do $\mathrm{pH}$ desejado de força Iônica 0,1 com uma soluçăo contendo uma con. centração conhecida (aproximadamente 85 $\mu \mathrm{M})$ de monóxido de carbono dissolvido em água. Após misturar, a concentração de hemoglobina, foi de aproximadamente $3 \mu \mathrm{M}$ em hemoequivalentes. A reação foi seguida a 420 e $435 \mathrm{~nm}$.

\section{Resultados}

Os hemolisados de Hoplosternum littorale aprêsentam duas bandas de hemoglobina em eletroforese de gel (Fig. 1). Os padrões foram idêniticos para todos os hemolisados. $0 \mathrm{com}$ - ponente mais anodal inclul $69 \%$ da hemoglobina total e apresenta uma mobilidade relativa de $0,52+0,02$ (Media + D.P.; $N=5$ ) comparada com albumina de soro de bovino. A hemoglobina humana $A$ apresentou mobilidade relativa de $0,66+0,01 \quad(N=5)$. A hemoglobina menos anódica é referida aqui como hemoglobina $\mathrm{I}$.

A focalização analítica isoelétrica do disco de gel de poliacrilamida indica que existem dois componentes de hemoglobina na conformação carboxi. Ab-I e Ab II apresentam pontos isoelétricos respectivamente reduzidos ou aumentados ao serem deoxigenadas. Isto indica

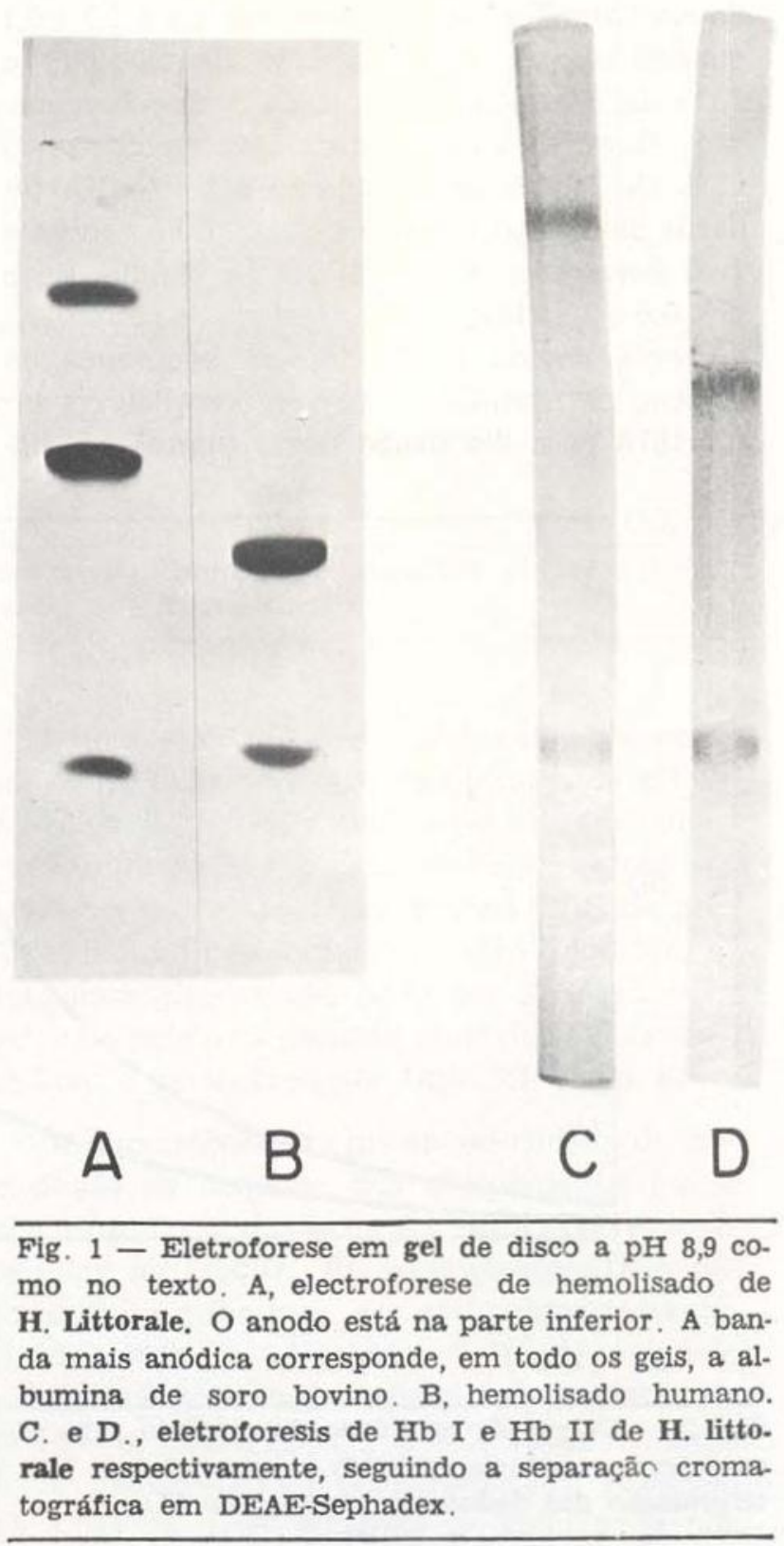


que $\mathrm{Hbl}$ deve ter um efelto Bohr inverso e $\mathrm{Hbll}$ um efeito Bohr normal (ver Bunn \& Riggs, 1978). Não foram observados tetrâmeros assimétricos híbridos de hemoglobina em geis de carboxihemoglobina ou de deoxihemoglobina .

O peso molecular das cadeias da hemoglobina de $H$. littorale foi de aproximadamente $14.000 \mathrm{em}$ eletroforese de gel SDS. Este valor é similar ao valor de 14.500 obtido pelo mesmo método para cadeias de hemoglobina humana

Os experimentos de equilíbrio de oxigênio no sangue total de $\mathrm{H}$. littorale indicam que a pressão parcial de oxigênio à saturação média $\left(P_{50}\right)$ a $30^{\circ} \mathrm{C}$ (temperatura aproximada da água do rio Solimões) é de $7,8 \mathrm{~mm} \mathrm{Hg}$ a pH $7,7+0,1$ quando uma mistura de $21 \%$ de oxigênio e $79 \%$ de nitrogênio é utilizada para reoxigenação. Quando uma mistura gasosa contendo $25 \% \mathrm{O}_{2}, 69,4 \%$ de nitrogênio e $5,6 \% \mathrm{CO}_{2}$ foi usada para reoxigenar as células, $\mathrm{o} \mathrm{P}_{50}$ aparente (ver Powers et al., 1978) foi de $17,8 \mathrm{~mm} \mathrm{Hg}$ a $\mathrm{pH} 6,6+0,1$ (Fig. 2). Usamos o termo $\mathrm{P}_{50}$ aparente devido a não termos segurança da posição da assíntota superior (ver Powers et al., 1978 para discussão deste ponto). A he- moglobina podia estar incompletamente saturada com oxigênio devido ao efeito Root.

A cromatografia em gel de DEAE Sephadex separa a hemoglobina de $\mathrm{H}$. littorale em dois componentes (Fig. 3). Eleroforese de cada fração separada (Fig. I) indica que cada uma é um componente puro. Dos $600 \mathrm{mg}$ de oxihemoglobina colocados na coluna, $180 \mathrm{mg}$ foram recuperados na primeira fração ( $\mathrm{Hbl}$ ) e $310 \mathrm{mg}$ foram recuperados na segunda (Hbll). Estes valores correspondem bem às proporções encontradas por eletroforese de gel de disco, 31 a $69 \%$ respectivamente.

A hemoglobina não fracionada tem um valor $\mathrm{P}_{50}$ de $3,8 \mathrm{~mm} \mathrm{Hg}$ a $\mathrm{pH} 7,0$ e $20^{\circ} \mathrm{C}$. O valor $P_{50}$ aumenta a $11,0 \mathrm{mmHg}$ na presença de $1 \mathrm{mM}$ ATP a pH 7,0. Apresentando efeito Bohr alcalino normal: $\Delta \log \mathrm{P}_{\mathrm{so}} / \Delta \mathrm{pH}$ parece máximo perto de $\mathrm{pH} 7,0$. A adição de $1 \mathrm{mM}$ ATP causa o máximo de efeito a $\mathrm{pH}$ de aproximadamente 7,2 (Fig. 4).

A hemoglobina não fracionada também apresenta efeito Root; porém a presença deste efeito não é tão forte quanto no cơmponente II purificado. O ATP aumenta o efeito Root em hemoglobina não fracionada.

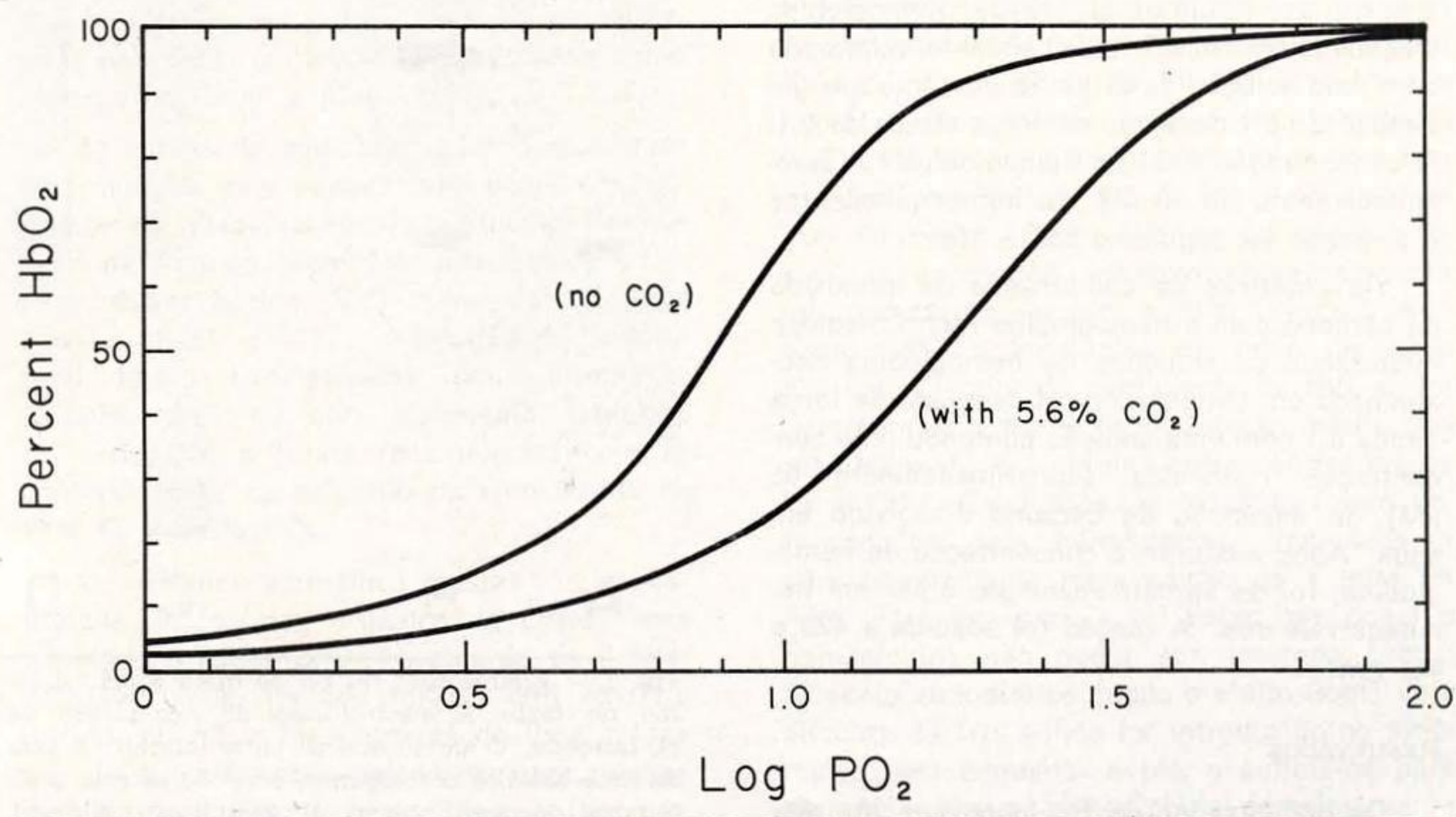

Fig. 2 - Cufrvas de equilibrio de oxigênio para o sangue total de H. littorale. As curvas representam a oxigenação em ausência ( $\mathrm{pH} 7,7$ ) e presença $(\mathrm{pH} 6,6)$ de $3,6 \% \mathrm{CO}_{2}$. Ver texto por precaução em relação à interpretagăo dos dados em presença de $\mathrm{CO}_{2}$. 


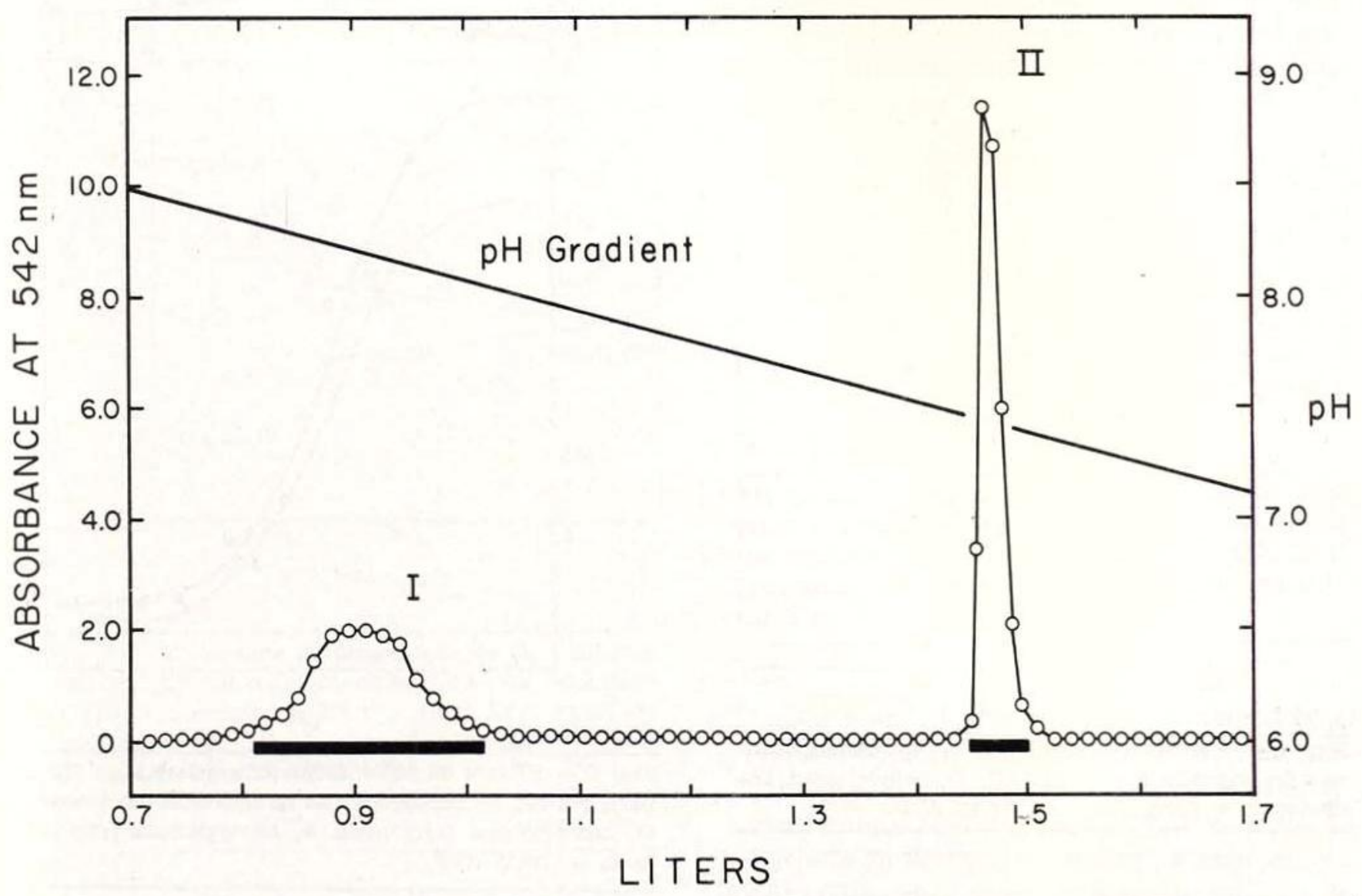

Fig. 3 - Cromatografia de DEAE-Sephadex da oxiemoglobina de H. littorale. As condições são as descritas no texto. As barras abaixo dos picos I e II indicam as frações que foram unidas.

Os equilíbrios de oxigênio das hemoglobinas I e II são notavelmente diferentes (Figs. $5,6)$. A pH $7,0\left(20^{\circ} \mathrm{C}\right)$ os valores de $P_{50}$ das duas hemoglobinas são $1,3 \mathrm{~mm} \mathrm{Hg}$ (fracionadas) e $6,3 \mathrm{~mm} \mathrm{Hg}$ (fracionadas $+1 \mathrm{mM}$ ATP) para $\mathrm{Hb}-\mathrm{I}$ e correspondendo ao valor de $\mathrm{P}_{50}$ de $8 \mathrm{~mm} \mathrm{Hg}$ e de $13 \mathrm{~mm} \mathrm{Hg}$ ( $1 \mathrm{mM} \mathrm{ATP)}$ para Hb-II fracionada a $\mathrm{pH} 7,0$ e $20^{\circ} \mathrm{C}$. Hb-I fracionada mostra um efeito Bohr inverso ou ácido na fai$x a$ de $\mathrm{pH} 6,0$ a $8,0\left(\Delta \log \mathrm{P}_{50} / \Delta \mathrm{pH} \cong+0,26\right)$ e um ligeiro efeito Bohr alcalino entre $\mathrm{pH} 8,0 \mathrm{e}$ $9,0\left(\Delta \log \mathrm{P}_{\mathrm{so}_{0}} / \Delta \mathrm{pH} \cong-0,12\right)$.

Hb-I não apresenta efeito Root, nem na condição fracionada, nem na presença $1 \mathrm{mM}$ ATP. Na presença de $1 \mathrm{mM}$, HB-I apresenta uma diminuição de 6 vezes na afinidade de oxigênio a $\mathrm{pH} 6,0$ e mostra um efeito Bohr alcalino normal entre $\mathrm{pH} 7,0$ e $8,0: \Delta \log \mathrm{P}_{\mathrm{so}} / \Delta \mathrm{pH}=-0,22$ (Fig. 5).
A hemoglobina II, por outro lado, apresenta um efeito Bohr normal forte na faixa de $\mathrm{pH}$ de 6,0 a $8,0: \Delta \log \mathrm{P}_{50} / \Delta \mathrm{pH}=-0,53$ para hemoglobina fracionada; o valor diminui a $-0,66 \mathrm{em}$ presença de $1 \mathrm{mM}$ ATP na mesma faixa de $\mathrm{pH}$. A pH baixo Hb-II apresenta efeito Root, isto é, esta hemoglobina não pode ser saturada com oxigênio pelo ar à pressão atmosférica. O efeito Root é aumentado por $1 \mathrm{mM}$ ATP (Fig. 6) .

A dependência de $\mathrm{pH}$ na velocidade de dissociação de oxigênio dos componentes I e II em presença e ausência de $1 \mathrm{mM}$ ATP é apresentada na Fig. 7. As hemoglobinas são notoriamente diferentes em seu comportamento. A constante de velocidade "off" de oxigênio para o componente I é quase independente do $\mathrm{pH}$. Na ausência do ATP, a velocidade aumenta ligeiramente com um aumento de $\mathrm{pH}$. A adição de $1 \mathrm{mM}$ de ATP aumenta a velocidade nos 


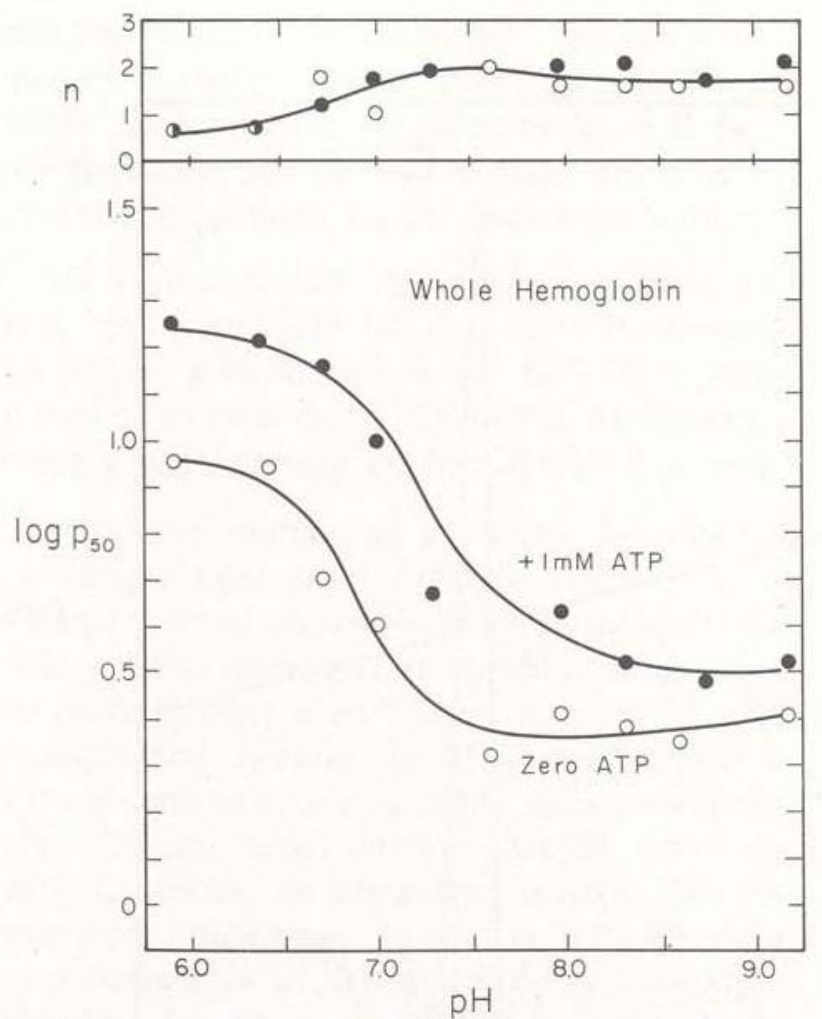

Fig. 4 - Valores de Log $P_{50}$ e " $n$ " de hemoglobina não fraccionada a diversos $\mathrm{pH}$. $\mathrm{O}$, hemoglobina fracionada. $\bullet$ hemoglobina $+1 \mathrm{~m}$ ATP.

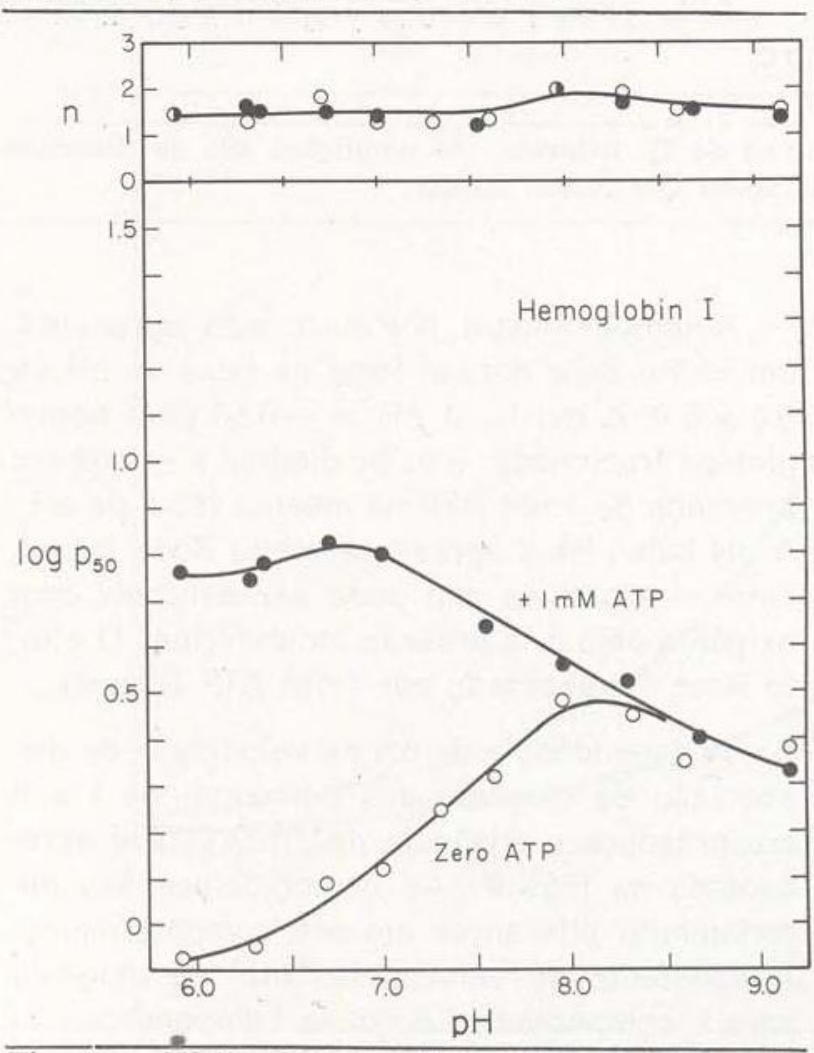

Fig. 5 - Efeito de pH e ATP sobre os valores de Log $P_{50}$ e " $n$ " para Hb-I. As condições são as do texto. $\mathrm{O}$, hemoglobina fracionada. $\bullet$, hemoglobina

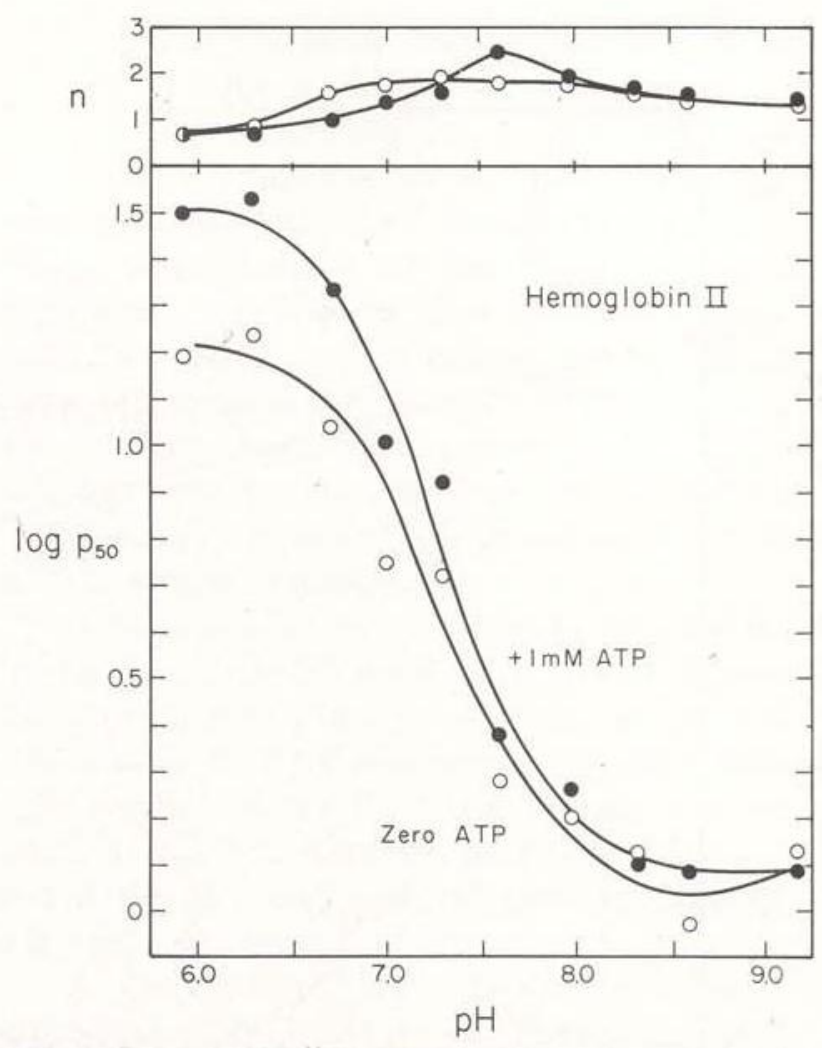

Fig. 6-Efeitos de pH e ATP sobre o Log $\mathrm{P}_{50}$ e " $\mathrm{n}$ " para Hb-II. As condições são as descritas no texto. O, hemoglobina fracionada. •, hemogiobina fracionada $+1 \mathrm{mM}$ ATP.

valores de $\mathrm{pH}$ baixos, mas não tem efeito a $\mathrm{pH} 8,3$, de maneira que a dependência global de $\mathrm{pH}$ é inversa do normal, além de pequena. Em contraste, a constante de velocidade "off" de $\mathrm{O}_{2}$ para o componente II é fortemente dependente do $\mathrm{pH}$ e aumenta umas 25 vezes quando o pH é diminuido de 8,0 a 5,7 na ausência de ATP. O ATP altera esta taxa, porém, sendo muito menos efetivo que os prótons. A adição de $1 \mathrm{mM}$ ATP aumenta a constante de velocidade em todos os valores de $\mathrm{pH}$ abaixo de 7,5 e aumenta a mudança com pH até umas 240 vezes.

A dependência da constante de velocidade de segunda ordem ( $\ell$ ') para a reação de monóxido de carbono com a componente de hemoglobina é apresentado na Fig. 8.

$\mathrm{Na}$ ausência de ATP $\ell^{\prime}$ diminui de $5 \times 10^{5} \mathrm{M}^{-1} \mathrm{~s}^{-1}$ a $\mathrm{pH} 5,4$ até aproximadamente $3 \times 10^{5} \mathrm{M}^{-1} \mathrm{~s}^{-1}$ a $\mathrm{pH} 8,0$. A adição de $1 \mathrm{mM}$ ATP diminui o valor de $\ell^{\prime}$ a aproximadamente $2 \times 10^{5} \mathrm{M}^{-1} \mathrm{~s}^{-1}$ entre $\mathrm{pH}$ 6 e 8 e elimina a dependência de pH. 


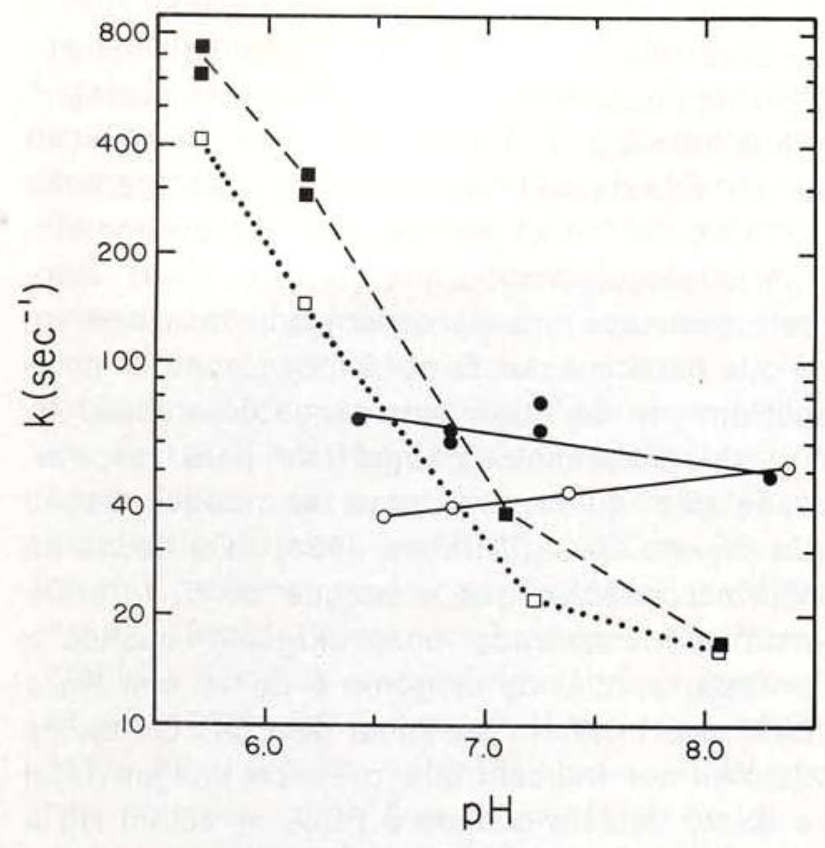

Fig. 7 - Velocidade de dissociação de $\mathrm{O}_{2}$ a diferentes $\mathrm{pH}$. " $\mathrm{K}$ " é a constante dissociação de velocidade. O, Hb-I fracionado. $\bullet$ Hb-I + $1 \mathrm{mM}$ ATP. $\square, \mathrm{Hb}$-II fracionado. $-\mathrm{Hb}-\mathrm{II}+1 \mathrm{mM}$ ATP. As condições são as indicadas no texto.

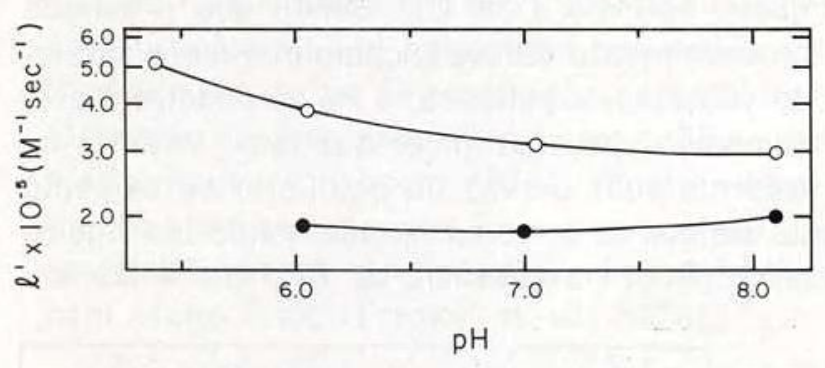

Fig. 8 - Velocidade de combinação de CO com $\mathrm{Hb}$ I a diversos $\mathrm{pH}$. L' é a constante da velocidade de combinação. O, Hb-I fracionada. $\bullet$ Hb-I fracionada + ImM ATP. As condições são indicadas no texto.

A dependência de $\mathrm{pH}$ de $\ell^{\prime}$ para o componente II da hemoglobina está expresso na Fig. 9. Tanto na presença quanto na ausência de $1 \mathrm{mM}$ ATP, a velocidade de reação tem uma dependência de $\mathrm{pH}$ bem diferente daquela apresentada pela hemoglobina I, aumentando mais de duas vezes a medida que o $\mathrm{pH}$ é aumentado de 6,0 a 8,0 . Parece que o ATP tem pouco efeito. Expressado como porcentagem do efeito total de $\mathrm{pH}$, o efeito é provavelmente semelhante ao do ATP na velocidade de dissociação de $\mathrm{O}_{2}$ para esta proteína, mas dada a dependência de $\mathrm{pH}$ tão reduzida na reação de combinação de $\mathrm{CO}$, o efeito de ATP é tão reduzido que se aproxima dos limites de confiança das medidas.

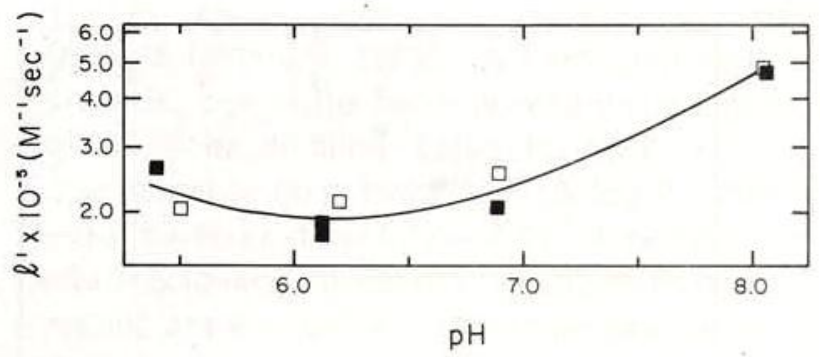

Fig. 9 - Velocidade de combinação de $\mathrm{CO}$ com Hb-II a diversos pH. L' é a constante de velocidade de combinação. $\square$, Hb-II fracionado. $\mathbf{n}$, Hb-II fracionado + $1 \mathrm{mM}$ ATP. As condições são as indicadas no texto.

As propriedades cinéticas do componente I da hemoglobina em ausência de ATP são tais que indicarn que sua atividade de ligação diminui com o aumento de $\mathrm{pH}$. A velocidade de dissociação de $\mathrm{O}_{2}$ aumenta com o aumento no $\mathrm{pH}$, sendo que a velocidade de combinação de CO diminui. Assim se poderia predizer um efeito Bohr inverso ou negativo em base a estes resultados. A adição de $1 \mathrm{mM}$ ATP inverte a dependência de $\mathrm{pH}$ da velocidade de combinação de $\mathrm{CO}$ (efeito Bohr positivo) .

As propriedades cinéticas do componente II da hemoglobina são totalmente diferentes. As duas constantes de velocidade variam com $0 \mathrm{pH}$ de uma maneira indicativa de um efeito Bohr normal. A extrema dependência da taxa de dissociação de $\mathrm{O}_{2}$ sugere que este efeito Bohr é, de fato, um efeito Root, isto é, que resulta em atividade tão baixa na faixa baixa de $\mathrm{pH}$ que impede a saturação da hemoglobina com oxigênio quando equilibrada com 0 ar. Sabe-se que este é o caso. Hemoglobina não fracionada e componente II apresentam efeito Root. Hb-1 não apresenta efeito Root. O efeito Root é incrementado com adição de $1 \mathrm{mM}$ ATP à hemoglobina fracionada completa ou hemoglobina II. A Fig. 10 indica que a $\mathrm{p} \mathrm{H7,0}$ a hemoglobina livre não fracionada e a hemoglobina com $1 \mathrm{mH}$ ATP estão ambas saturadas 
$100 \%$ com oxigênio ao $\mathrm{PO}_{2}$ do ar. $\mathrm{A}$ pH 6,7 a hemoglobina fracionada é completamente saturada mais com $1 \mathrm{mM}$ ATP está só $88 \%$ saturada. A . pH 5,9 a hemoglobina livre não fracionada está $73 \%$ saturada como oxigênio e a hemoglobina com $1 \mathrm{mM}$ ATP está $69 \%$ saturada.

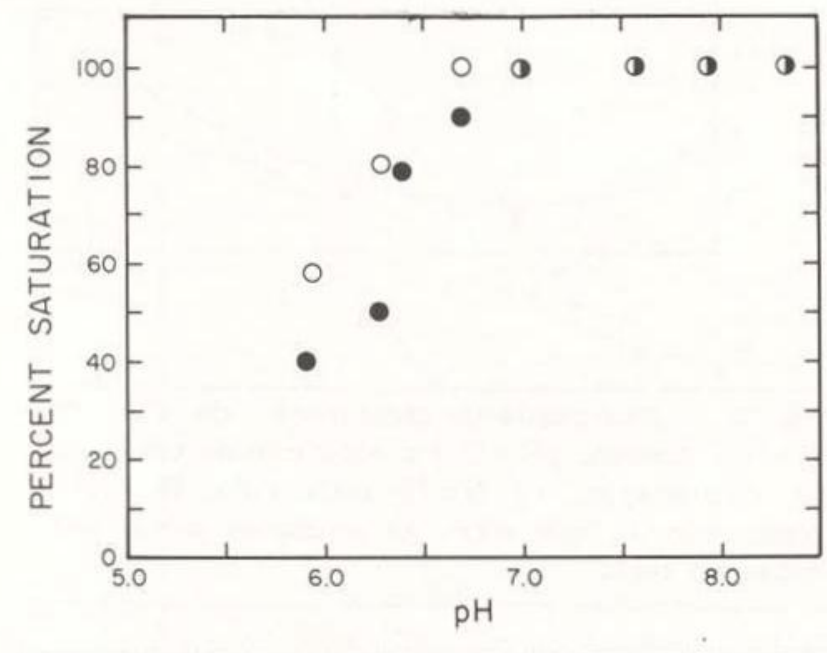

Fig. 10 - Curva de transição do efeito Root para hemoglobina não fracionada. A percentagem de saturação com oxigênio atmosférico está dada a diversos $\mathrm{pH}$. O, hemoglobina fracionado. $\bullet$, hemoglobina fracionado $+1 \mathrm{mM}$ ATP.

A hemoglobina II apresenta uma curva de transição do efeito Root muito semelhante à da hemoglobina completa em que a percentagem de saturação começa a diminuir a valores de $\mathrm{pH}$ abaixo de 7,0, mas o declive é maior para $\mathrm{Hb}$-ll que para hemoglobina completa (Fig. 11). A pH 5,9, a hemoglobina II fracionada está $58 \%$ saturada com oxigênio a pressão atmosférica, sendo que só está $40 \%$ saturada nas mesmas condiçōes em presença de $1 \mathrm{mM}$ ATP.

\section{DISCUSSÃo}

O bagre Hoplosternum littorale tem-se adaptado à vida em águas hipóxicas evoluindo um intestino altamente vascularizado, com o qual respira oxigênio atmosférico (Carter \& Beadle, 1931). Este peixe é um respirador obrigatório de ar cujas guelras sảo consideradas atualmente "como auxiliares na eliminação de dióxido de carbono em vez de na procura de oxigênio (Willmer, 1934). Willmer, (1934) usando o método de van Slyke concluiu que o sangue total de $\mathrm{H}$. littorale apresenta uma afinidade de oxigênio moderadamente baixa e apresenta um efeito Bohr em resposta à adiçăo de dióxido de carbono pequeno em comparação com os de outros peixes examinados por ele. $\mathrm{Em}$ adição, observou que o sangue de $H$. littorale tem uma grande capacidade de oxigênio, o que parece estar correlacionado com o comportamento de respiração aérea do animal. $H$. littorale deve sair à superfície para respirar cada 10 minutos, sem importar a concentração de $\mathrm{CO}_{2}$ da água (Willmer, 1934). Os dados de Willmer indicam que o sangue de $\mathrm{H}$. littorale está meio saturado com oxigênio quando a pressão parcial de oxigênio é de $10 \mathrm{~mm} \mathrm{Hg}$ a $28^{\circ} \mathrm{C}$ e pH 6,8 em ausência de $\mathrm{CO}_{2}$. Os dados de Wilimer indicam que o $P_{50}$ do sangue total é de $20 \mathrm{~mm} \mathrm{Hg}$ quando $\circ \mathrm{PCO}_{2}=25 \mathrm{~mm} \mathrm{Hg}$ a $\mathrm{pH} 5,8\left(28^{\circ} \mathrm{C}\right)$. Nossos dados, obtidos usando o analisador de dissociação de oxigênio Hem$\mathrm{O}$-Scan indicam que a $30^{\circ} \mathrm{C}$ o $\mathrm{P}_{50}$ para o sangue total de $\mathrm{H}$. littorale é de $7,8 \mathrm{~mm} \mathrm{Hg}$ na ausência de $\mathrm{CO}_{2}$ e a pH 7,7 a $30^{\circ} \mathrm{C}$ o $\mathrm{P}_{50}$ aparente é de $17,8 \mathrm{~mm} \mathrm{Hg}$ quando $42 \mathrm{~mm} \mathrm{Hg}$ de $\mathrm{CO}_{2}$ são agregados $\left(\mathrm{pH} 6,6\right.$ a $\left.30^{\circ} \mathrm{C}\right)$. Sendo que o sangue provavelmente estava incompletamente saturado sob estas condições, o $\mathrm{P}_{50}$ verdadeiro é presumivelmente algo maior que isto. Willmer interpreta suas curvas de equilíbrio de oxigênio de sangue total como apresentando um ligeiro efeito Root na ausência de $\mathrm{CO}_{2}$ que é aumen-

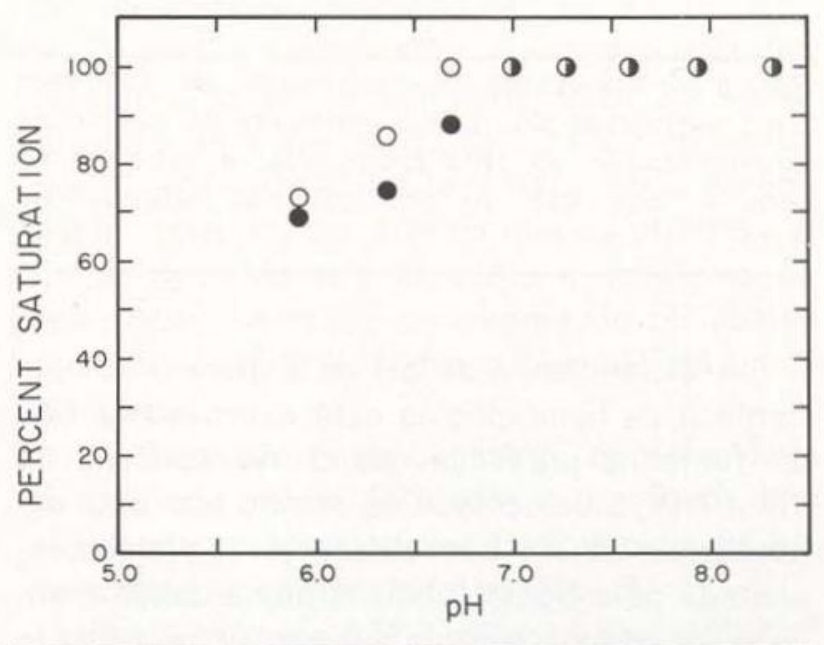

Fig. 11 - Curva de transiçăo do efeito Root para Hb-II fracionada. A percentagem de saturação com oxigênio atmosférico está dada a diversos $\mathrm{pH}$. $\mathrm{O}$, Hb-II fracionada. $\bullet$ Hb-II fracionada $+1 \mathrm{mM}$ ATP. 
tado com adição de $\mathrm{CO}_{2}$. Esta conclusão depende de uma extrapolação relativamente grande de pressões entre mais ou menos $35 \mathrm{~mm} \mathrm{Hg}$ a $150 \mathrm{~mm} \mathrm{Hg}$. Nossos dados indicam que a hemoglobina diluída e não fracionada de $H$. littorale apresenta um pequeno efeito Root $\mathrm{e}$ que um componente da hemoglobina ( $\mathrm{Hb}-\mathrm{II}$ ) porém não os outros, apresenta um efeito Root considerável. O efeito Root em $\mathrm{Hb}-\mathrm{Il}$ de $\mathrm{H}$. littorale é comparável àqueles encontrados em certas outras hemoglobinas de peixes. A hemoglobina da carpa (Cyprinus carpio) só está meio saturada a $\mathrm{pH} 5,5$ e $20^{\circ} \mathrm{C}$ na presença de fosfatos orgânicos endógenos (Noble et al., 1970). Certos componentes das hemoglobinas das duas trutas Salmo irideus (Brunori et al., 1973) e S. gairdneri (Lan et al., 1975) estão só uns $20 \%$ saturados com o oxigênio nas mesmas condições. A perda de oxigênio por certas hemoglobinas de peixe equilibradas a $\mathrm{pH}$ baixo com oxigênio atmosférico (efeito Root) tem sido tida como parte do mecanismo pelo qual o oxigênio é secretado na bexiga natatória para manter ou controlar a quantidade de flutuação neutra. Não é sabido se a hemoglobina com efeito Root de $\mathrm{H}$. littorale cumpre este propósito. Até agora não havia sido encontrado um peixe de respiração aérea obrigatória que apresentasse uma hemoglobina com efeito Root (Johansen, 1970). Porém, atualmente, tem-se encontrado outros peixes de respiração aérea que possuem hemoglobinas com efeito Root (Farmer et al., 1978).

O componente II da hemoglobina de $H$. littorale aparentemente liga $\mathrm{o}_{2}$ de maneira não cooperativa a pH baixo. Este componente é similar à da hemoglobina de carpa (Tan et al., 1973) e da fração 3 da hemoglobina de truta (Lan et al., 1975). Ainda se um valor de $n=1$ pode refletir ausência de cooperatividade nos dados de $\mathrm{H}$. littorale, podia também resultar da ligação de oxigênio a subunidades funcionalmente diferentes subunidades $\propto$ e $\beta$. A queda de $\mathrm{n}$ a $\mathrm{pH}$ baixo é aumentada por ATP em medições feitas com $\mathrm{Hb}-\mathrm{Il}$ de $\mathrm{H}$. littorale. A falta aparente de cooperatividade à afinidade mínima de ligação em pH suficientemente baixo está associado com o efeito Root e pode ser desčrita qualitativamente pelo menos no "estado $T^{\prime \prime}$ do modelo alostérico de Monod et al. (1965).
A adição de $1 \mathrm{mM}$ ATP à Hb-I de $H$. littorale muda o efeito Bohr inverso a um efeito Bohr, alcalino normal. Em $\mathrm{Hb}$ A humana e em certas outras hemoglobinas de mamíferos, o efeito Bohr inverso ou ácido, presente a $\mathrm{pH}$ baixo, é aumentado com adição de fosfatos orgânicos (Bailey et al., 1970; De Bruin et al., 1971; Riggs \& Imamura, 1972). A hemoglobina II fracionada, por outro lado, apresenta um grande efeito Bohr alcalino $\left(\Delta \log \mathrm{P}_{50} / \Delta \mathrm{pH}=-0,8\right)$ que aumenta com $1 \mathrm{mM}$ ATP $\left(\Delta \log \mathrm{P}_{50} / \Delta \mathrm{pH}=\right.$ $-1,0$ na faixa de $\mathrm{pH} 7,0-7,5)$. A hemoglobina não fracionada apresenta um efeito Bohr muito menor entre $-0,4$ e $-0,5$ como era de se esperar.

Os hemolisados de certos peixes apresentam componentes múltiplos de hemoglobina, nos quais as propriedades de cada componente são notoriamente diferentes umas das outras (Binotti et al., 1971; Powers \& Edmundson, 1972; Gillen \& Riggs, 1973; Weber et al., 1976). As hemoglobinas são geralmente de dois tipos (discutido em Weber et al., 1976); uma apresenta afinidade de oxigênio relativamente alta e é relativamente inalterada pelo $\mathrm{pH}$. O outro tipo de hemoglobina tem uma afinidade de oxigênio menor, mostra o efeito Bohr alcalino normal e apresenta efeito Root. Concomitante com a evolução do efeito Root, há um efeito potencialmente indesejável. Uma perda grande de oxigênio do sangue da bexiga natatória pode trazer um grau perigoso de hlpoxia intolerável para o peixe. Certos peixes parecem solucionar este problema sintetizando duas hemoglobinas funcionalmente diferentes; uma possuindo um efeito Root, sendo que a outra liga o oxigênio independente do $\mathrm{pH}$ (Riggs, 1976). Powers \& Edmundson (1972) sugerem que as hemoglobinas de efeito Bohr nulo podem ser úteis em evitar a anóxia resultante da perda da ligação de oxigênio pela hemoglobina de efeito Root que pode seguir a produção de ácido lático após de exercícios viơlentos de emergência. É concebível que a hemoglobina I de $\boldsymbol{H}$. littorale com seu forte efeito Bohr inverso pode efetivamente cumprir tal propósito. Além disto, a hemoglobina I pode atuar como um depósito parcial de oxigênio durante os 10 minutos que o peixe permanece submerso. A presença de uma hemoglobina de efeito Bohr inverso neste peixe 
pode servir para modular a hemoglobina de efeito Root numa situação de $\mathrm{pH}$ baixo tal como durante uma imersão onde há acúmulo de $\mathrm{CO}_{2}$. Bonaventura et al., (1977) sugerem que a hemoglobina de efeito Bohr inverso do anfíbio Amphiuma means pode ser especialmente útil durante a Invernação ou estivação. Não é sabido se $H$. littorale pode estivar, mas achamos que certas pequenas lagoas contendo este peixe estavam no processo de secamento sendo que outras lagoas semelhantes já haviam secado. Restos de várias espécies de peixes, incluindo $H$. littorale encontrados nestas lagoas secas sugerem que esta espécie morre nessas condições. Johansen, (1970) cita a estivação como uma maneira na qual os peixes tropicais podem combater a anóxia e dissecação. Ele anota que o peixe pulmonado estiva quando as lagoas ou os igarapés secam na Africa. Se Hoplosternum estiva o meio ambiente pode virar hipóxico e acídico por acúmulo de $\mathrm{CO}_{2}$. Sob estas condições. uma diminuição no conteúdo de fosfato orgânico dos glóbulos vermelhos (ver Wood \& Johansen, 1972) podem auxiliar à $\mathrm{Hb}-\mathrm{I}$ a atuar como um reservatório mais eficiente de oxigênio. De qualquer maneira, a presença de duas hemoglobinas distintas afetadas de diferentes maneiras por fosfatos orgânicos presumivelmente permitem a $\mathrm{H}$. littorale um controle mais amplo para enfrentar a mudança de disponibilidade de oxigênio durante épocas secas e úmidas alternas.

\section{AgRADECIMENTOS}

Este trabalho foi apoiado pela bolsa PCM06451 da National Science Foundation para estudos a bordo do R/V "Alpha Helix". Agradecemos aos brasileiros a colaboração e ao terem possibilitado que o R/V "Alpha Helix" entrasse no rio Solimões. Queremos agradecer ao Capitão Clarke e a tripulação pela cooperação. Apoio adicional foi dado pelo ConseTho de Pesquisas para Ciências e Humanidades da Noruega (H.J. e U.E.H.F.), bolsa NSF DEB-76-79877 (D.A.P.), a National Geographic Society (D.A.P.), bolsa NIH HL-15460 (A J. Bonaventura para apoio de J.P.M. ), bolsa NSF PCM - 76-06719 (A. Riggs), bolsa NIH GM21314 (A. Riggs) e pela Fundação Nehemias Gorin (a H.F. Bonn).

\section{SUMMARY}

The two hemoglobins, I and II of the obligate air-breathing catfish, Hoplosternum littorale have been isolated. The unfractionated stripped hemoglobin has a high oxygen affinity, a normal alkaline Bohr effect, and a Root effect. Both the Bohr and Root effects are enhanced by $1 \mathrm{mM}$ ATP. Stripped hemoglobin 1 has a relatively high oxygen affinity a reversed Bohr effect bethween $\mathrm{pH} 6.0$ and $8.0\left(\Delta \log \mathrm{P}_{50} / \Delta \mathrm{pH}>0\right)$ but no Root effect. Addition of $1 \mathrm{mM}$ ATP to $\mathrm{Hb}$ I causes a marked reduction in the oxygen affinity, a change to a normal alkaline Bohr effect $\left(\Delta \log P_{50} / \Delta p H>0\right)$, but no Root effect. Stripped hemoglobin II has a lower oxygen affinity at low $\mathrm{pH}$ and a higher oxygen affinity at high $\mathrm{pH}$ than does hemoglobin I. Hemoglobin II shows a large alkaline Bohr effect which is only slightly increased by $1 \mathrm{mM}$ ATP and a Root effect at low pH which is enhanced by $1 \mathrm{mM}$ ATP. The observed rates of $\mathrm{O}_{2}$ dissociation and of $\mathrm{CO}$ combination with components I and II show differences which parallel those observed in the oxygen equilibrium measurements.

\section{BIBLIOGRAFIA}

Bailey, J. E.; Beetlestone, J. G. \& Irvine, D. H.

1970 - Reactivity differences between hemoglobins. Part XVII. The variability of the Bohr effect between species and the effects of 2,3diphosphoglyceric acid. J. Chiem. Soc. London (A) : 756-762.

Binotti, I.; Giovenco, S. Giardina, B.; Antonini, E.; BRUNORI, M. \& WYMAN, J.

1971 - Studies on the functional properties of fish hemoglobins. II. The oxygen equilibrium of the isolated hemoglobin components from trout blood. Arch. Biochem. Biophys, 142: 274-280.

Bonaventura, C. ; Sullivan, B. \& Bonaventura, J. \& BOURNE, $\mathrm{S}$.

1977 - Anion modulation of the negative Bohr effect of hemoglobin from a primitive amphibian. Nature, 265, 474-476.

Brunori, M.; Bonaventura, J. \& Bonaventura, C.

Giardina, B.; Bossa, F. \& ANTONINI, E.

1973 - Hemoglobin from the trou: structural and functional properties. Molec. cell. Biochem., 1: 189-196.

BUNN, H. F. \& RIGGS, A.

1978 - A medida do efeito Bohr em hemoglobinas de peixe por focalização elétrica em gel. Acta Amazonica $8(4)$ : Suplemento. (Este volume).

Carter, G. S. \& Beadle, L. C.

1931 - The fauna of the swamps of the Paraguayan Chaco in relation to its environment. II. Respiratory adaptations in the fishes. J. Linnean Soc. (London), 37: 327-366. 
De Bruin, S . H; JANSSEN, L. H. M. \& VAN Os, G, A. J. 1971 - Effect of 2,3-diphosphoglycerate on the Bohr effect of human adult hemoglobin. Biochem. Biophys. Res. Commun., 45 : 544-550.

FARMER, M.; FYHN, H. J.; FYHN, U. E. H.

\& NOBLE, R. W.

1978 - Ocorrência de hemoglobinas de efeito Root em peixes amazônicos. Acta Amazonica 8 (4) : Suplemento. (Este volume).

FYhN, U. E. H.; FYHN, H. J.; DAvis, B. J.; Powers, D. A.; FInK, W. L. \& Garlick, R. L.

1978 - Heterogeneidade de hemoglobina nos pelxes da Amazônia. Acta Amazonica $8(4)$ : Suplemento. (Este volume).

Gibson, Q. H. \& MILNES, L.

1964 - Apparatus for rapid and sensitive spectrophotometry. Blochem. J., 91: 161-171.

GILlen, R. G. \& Rigos, A.

1973 - Structure and function of the isolated hemoglobins of the America eel, Anquila rostrata. J. Biol. Chem., 248 : 1961-1969.

JOHANSEN, K

1970 - Air breathing In fishes. In : Flsh Physlolo. gy, v. 4, New York, Academic Press, pp. 361.411.

Kramer, D. L. \& Graham, J. B.

1976 - Synchronous air breathing, a soclal component of respiration in fishes. Copela, 689-697.

lau, H. K. F.; Wallach, D. E.; Pennelly, R. R.

\& NOBLE, R. W.

1975 - Ligand blnding propertles of hemaglobln 3 of the trout Salmo galrdneri: The occurrence of an acid Bohr effect in the absence of heme-heme interactions. J. Blol. Chem. 250: 1400-1404.

Monod, J.; Wyman, J. \& Changeux, J.

1965 - On the nature of allosteric transitions : plausible model. J. Mol. Biol., 12: 88-118.

Noble, R. W.; PARKhurst, L. J. \& Gibson, Q. H. 1970 - The effect of $\mathrm{pH}$ on the reaction of oxygen and carbon monoxide with the hemoglobln of the carp, Cyprinus carpio. J. Blol. Chem., 245 : 6628-6633.

Powers, D. A. \& Edmundson, A. B.

1972 - Multiple hemoglobins from catostomld flsh. 1. Isalotion and characterization of the Isohemoglobins of Catostomus clarkll. J. Biol. Chem., 247 : 6686-6693.

POWers, D. A.; FyHN, H. J.; FyHN, U. E. H.;

Martin, J. P.; GArllick, R. L. \& WoOd, S. C.

1978 - Estudo comparativo de equilibrio de oxigênio no sangue de 40 gêneros de peixes da Amazônia. Acta Amazonia 8(4) : Suplemento. (Este volume).

Riggs, A.

1976 - Factors in the evolution of hemoglobin function. Fed. Proc., $35:$ 2115-2118.

Riggs, A. \& ImAMURA, T.

1972 - Enhancement of the acid and alk Inne Bohr effects of hemoglobin by organic phosphates. Adv. Exp. Med. Blol., 28 : 55.63.

Riggs, A. \& Wolbach, R. A.

1956 - Sulfhydryl groups and the structure of hemoglobin. J. Gen. Physiol., 39 : 585-605.

Tan, A. L.; Noble, R. W. \& Gibson, Q. H.

1973 - Conditions restrincting allosteric transitions In carp hemoglobin. J. Biol. Chem., 248: 2880-2888.

Weber, R. E.; Lykkeboe, G. \& Johunsen, K.

1976 - Physiological propertles of eel hemoglobln : hypoxic acclimation, phosphate effects, and multiplicity. J. Exp. Biol., $64:$ 75-88.

WILLMER, E. M.

1934 - Some observations on the respiration of certain tropical fresh-water fishes. J. Exp. Biol., 11: 283-306.

WOOD, S. C. \& JohANSEN, K.

1972 - Adaptation to hypoxia by increased $\mathrm{HbO}_{2}$ affinlty and decreased red cell ATP concentration. Nature New Biology, 237: 278-279 